\title{
Erratum: Single-Step Step Ladder Expansive Cranioplasty
}

\author{
S. K. Sengupta ${ }^{1}$ \\ ${ }^{1}$ Command Hospital (EC), Kolkata, West Bengal, India \\ Indian J Neurosurg 2017;6:e2.
}

\author{
Address for correspondence Col. S. K. Sengupta, MS (Gen Surg), \\ MCh (Neurosurgery), 349/2; Dakshin Behala Road, Kolkata, \\ West Bengal 700061, India \\ (e-mail: sudipkumarsengupta14@rediffmail.com).
}

\section{ERRATUM}

The article by Dr. S. K. Sengupta published online on May 19, 2017 (doi: 10.1055/s-0037-1602755) was originally published with the title "Single-Step Step Ladder Expansive Cranioplasty: A Case Report". There is an update to the article title. The updated title is "Single-Step Step Ladder Expansive Cranioplasty". 